\title{
EFFECTIVE SAR SEA ICE IMAGE SEGMENTATION AND TOUCH FLOE SEPARATION USING A COMBINED MULTI-STAGE APPROACH
}

\author{
Jinchang Ren ${ }^{1}$, Byongjun Hwang ${ }^{2}$, Paul Murray ${ }^{l}$, Soumitra Sakhalkar ${ }^{1}$, and Samuel McCormack ${ }^{1}$ \\ jinchang.ren@strath.ac.uk, Phil.Hwang@sams.ac.uk \\ ${ }^{1}$ Department of Electronic and Electrical Engineering, University of Strathclyde, Scotland, U.K. \\ ${ }^{2}$ Scottish Association for Marine Science, Oban, Scotland, U.K.
}

\begin{abstract}
Accurate sea-ice segmentation from satellite synthetic aperture radar (SAR) images plays an important role for understanding the interactions between sea-ice, ocean and atmosphere in the Arctic. Processing sea-ice SAR images are challenging due to poor spatial resolution and severe speckle noise. In this paper, we present a multi-stage method for the sea-ice SAR image segmentation, which includes edge-preserved filtering for preprocessing, k-means clustering for segmentation and conditional morphology filtering for post-processing. As such, the effect of noise has been suppressed and the undersegmented regions are successfully corrected.
\end{abstract}

Index Terms - Sea ice image segmentation; satellite synthetic aperture radar; edge-preserved filtering; k-means clustering; conditional morphology filtering; remote sensing.

\section{INTRODUCTION}

Sea-ice floe size distribution (FSD) is an important sea-ice parameter [1]. In winter sea ice normally forms continuous sheet of ice without apparent distinctive floes. As spring/summer melting approaches, the sheet of ice starts to be broken up into distinctive floes, mainly due to winds and ocean waves/swells. The degree of fragmentation of sea ice is not only dependent on external forcings (such as winds and waves) but also sea-ice properties itself (such as ice thickness and type). Rapid fragmentation of sea ice is expected to accelerate melting and summer sea ice minimum, yet more quantitate analysis of sea-ice fragmentation processes is still lacking, mainly due to very limited FSD data available from satellite data.

As optical and infrared sensors suffer from illumination and other environmental conditions, satellite synthetic aperture radar (SAR) has been widely used in monitoring sea ice as they are not limited by those factors. One of the primary reasons is that SAR is able to see through cloud and darkness, thus has been widely applied in a number of remote sensing applications. In this context accurate retrieval of FSD from SAR images is vital [2]. However, sea-ice SAR image segmentation has been always a challenging problem, mainly due to the limited spatial resolution, severe speckle noise and lack of features.

Various approaches have been proposed for SAR sea ice segmentation, such as image thresholding [2], data clustering [3], watershed and its variations [4, 10], edge-based techniques [5], feature fusion and learning [6], region growing [1, 7-9] and kernel graph-cut [11]. For threshold-based approaches, local/global thresholds are derived from histogram analysis for image segmentation. However, the segmented results can be severely affected by the presence of noise in the SAR images, which is also a common problem for edge based approaches. For feature and clustering based approaches, including region-growing, graph cut and watershed, they are less affected by noise as they consider more the local consistency of the pixels. However, due to inconsistency of reginal distribution, they may lead to under segmentation or over-segmentation of the images.

In summary, due to the challenging problems in terms of poor spatial resolution and heavy speckle noise, existing techniques show drawbacks and cannot deal properly the data for accurate segmentation of these SAR sea ice images, especially in detecting the boundaries and separating touch floes. As such, a multi-stage approach is proposed and presented in the paper to address these problems.

\section{THE APPROACH}

The proposed multi-stage processing approach include the following three key stages: pre-processing, clustering based segmentation, and conditional morphological filtering as postprocessing. As most SAR images contain the multiplicative noise known as speckle, the pre-processing step involves filtering the image using adaptive filters to remove the speckle noise before segmentation. We have used the adaptive median (AM) filter for this purpose, where local statistics within a filter window is used to mark a pixel as speckle noise and remove/reduce the speckle noise present in an image. As in general median filtering can remove the noise whilst preserving the edges, it can significantly improve the effectiveness of segmentation. We have compared our results with other popular speckle filtering techniques such as the Lee filter and Bilateral filter, and found that the AM filter is one of the most efficient techniques yet it can produce results comparable to other more complicated filters.

For image segmentation, K-means based pixel clustering is employed for its popularity as an unsupervised data clustering technique. The algorithm partitions/clusters a given set of data into $\boldsymbol{k}$ clusters depending on the least squared distance of each point from that cluster's centroid. K-means is an iterative process, which continually estimates the least squared distance from the cluster centroid and re-assigns the data into these ' $k$ ' clusters until the process is stabilized.

In our case, we simply set $k=2$, which correspond to sea ice and the water background, respectively. When a boundary can be clearly defined in the image, i.e. the contrast between two regions that should be separated are high, the segmentation results are 
satisfactory. However, due to the variations in terms of intensity and effect of noise, the results from K-means can be poor when the contrast of the adjacent regions is low. As a result, this has led to under-segmentation problem, where sea ice regions cannot be separately segmented from the water background. Another situation is the failure segmentation of two sea ice regions when the water boundary between them is thin, due to limited degree of melting.

To address these under-segmentation problems, morphological filtering is usually utilized to further separate sea ice floes $[1,3]$. However, applying morphological filtering to the segmented images can degrade the detected boundaries due to the opening and
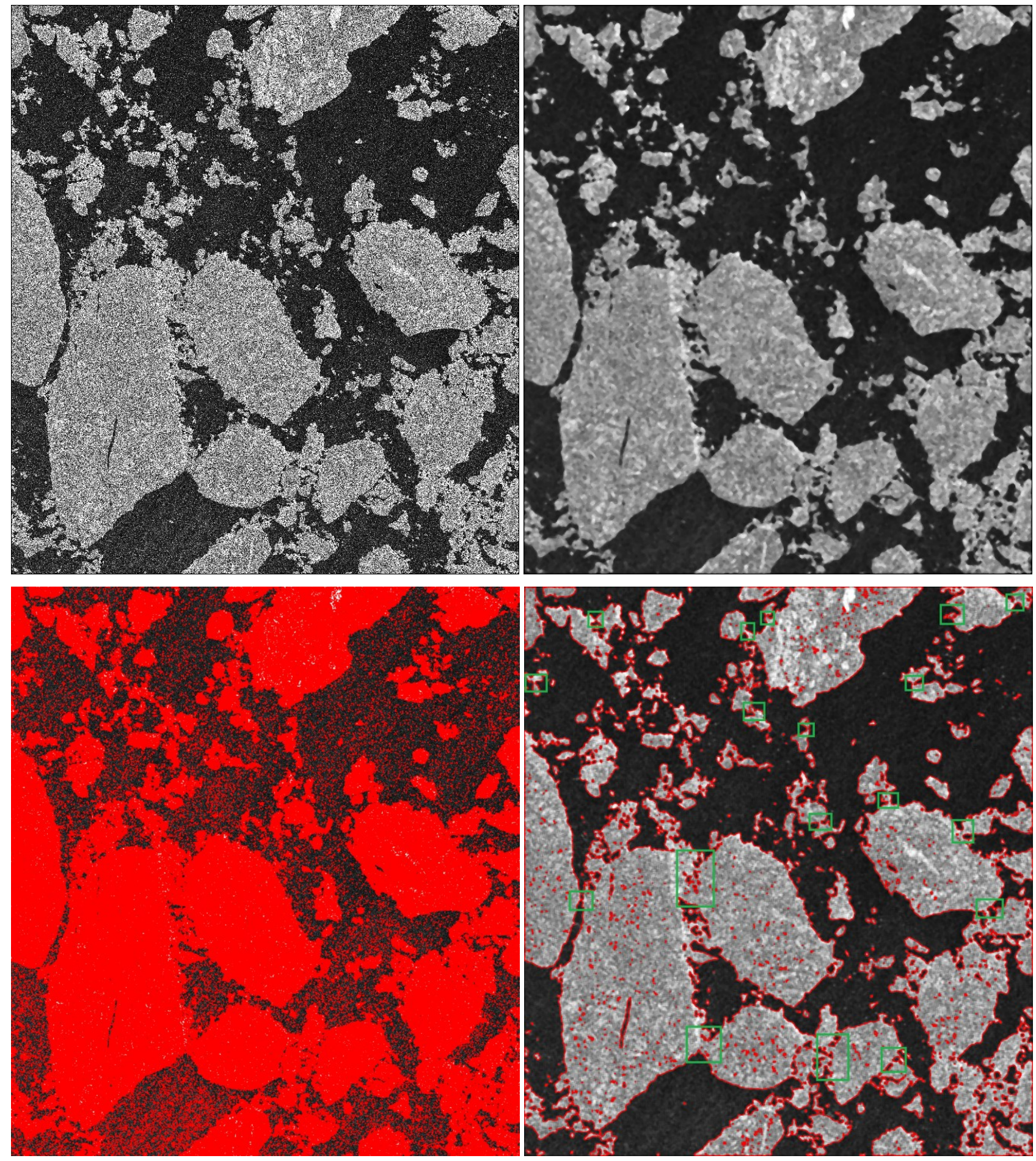

Fig. 1. One original image (top-left, (C DLR), the result after our median filtering based pre-processing (top-right) and the segmented results using $\mathrm{k}-$ means $(\mathrm{k}=2)$ (the bottom row). 
closing operations used and result in inaccurate floe size distribution analysis. To this end, we propose to use conditional morphological filtering where the opening and closing are only applied to the identified candidate areas of the segmented regions rather than onto the whole image.

Actually, some assumptions are used to identify such candidate areas: one is the concave property along the detected boundary, where a single sea ice block should be convex otherwise it may indicate under-segmentation of two sea ice regions. Another assumption is the size, where short boundary between two large sea ice regions can indicate possible under-segmentation, which is further identified using distance transform. Afterwards, based on the size and even orientation of the detected short boundary, a morphological filter is implemented adaptively and applied to the candidate area to further segment the image. This has successfully avoided distorting the segmented results and also saved the vast of processing time. Relevant results are reported in the next section for comparisons.

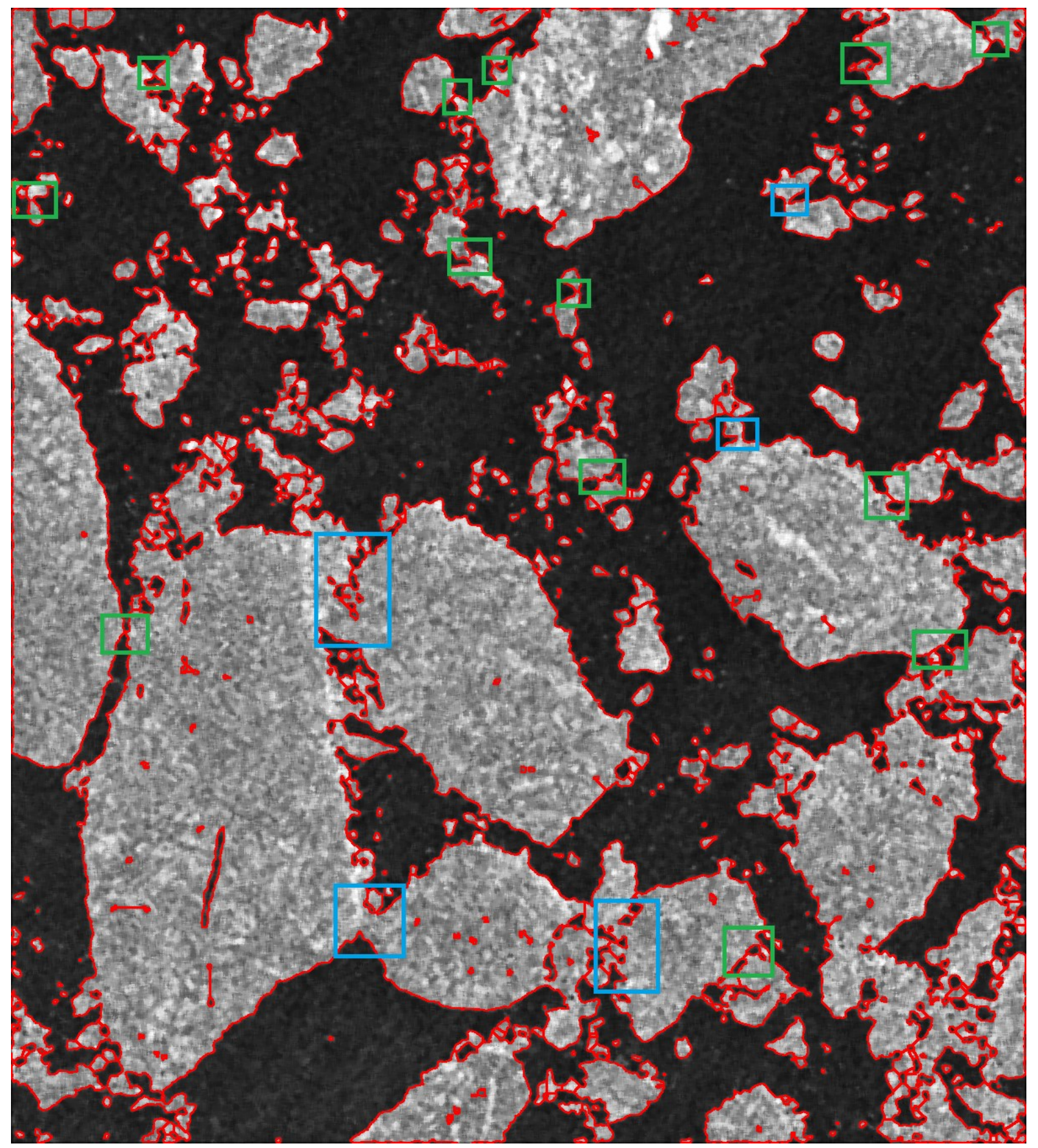

Fig. 2. Correction of under-segmented regions using conditional morphological filtering as highlighted in the green boxes, where blue boxes refer to those fail to be corrected. 


\section{EXPERIMENTS AND RESULTS}

The image we used was selected for TerraSAR-X single-pol StripMap (SM) image, taken on 2 August, 2014 (16:58 UTC). The pixel size is $1.25 \mathrm{~m} / \mathrm{pixel}$ and the full image size is about $25 \mathrm{k}$ by $48 \mathrm{k}$ pixels. The bit depth has been converted to 16 though the original intensity is up to $464 \mathrm{k}$ (about 19bits). For the effect of better visualization, results from a small portion of the image are shown in this section to illustrate how the proposed algorithm works.

Fig. 1 gives one original image and the results after preprocessing and corresponding segmented results using k-means. For easy comparison, the boundaries extracted from segmented regions are overlaid on the grey-scale image for comparison. As can be seen, due to severe noise, from the unfiltered image we cannot produce any meaningful segmentation. However, simply using the median filter has significantly improved the results of segmentation where most boundaries have been extracted. However, there are quite a few under-segmented areas as highlighted in Fig. 1 (see in the bottom-right image).

To deal with the under-segmentation problem and separation of touch floes, we applied the conditional morphological filtering as post-processing, and the results are shown in Fig. 2. As can be seen, most touch floes have been successfully separated by the introduced post-processing stage without damaging the accuracy of other boundaries. However, there are still some touch floes that cannot be separated, which are either due to the large gap between the touch floes or a number of melting ponds in between them. How to deal with such more complicated cases will be further investigated in the future.

\section{CONCLUSIONS}

A multi-stage approach is proposed in this paper for SAR sea ice segmentation and touch floe separation. With adaptive median filtering used as pre-processing, speckle noise has been suppressed with edges preserved. Using K-means for pixel clustering based segmentation, the results are generally satisfactory though having difficulty in dealing with regions of low contrast between water and sea ice. Finally, the proposed conditional morphological filtering has successfully separated touch floes and overcome the under-segmentation problem for more accurate segmentation and floe size distribution analysis of SAR sea ice images. Future work for more accurate segmentation is to combine several new approaches such as sparse representation [12], deep learning $[13,14]$ and singular spectrum analysis $[14,15]$.

\section{ACKNOWLEDGEMENTS}

TerraSAR-X data was obtained through DLR TSX SSS project (OCE2306). This work is partly funded through the grants (NE/L012707/1) and (NE/M00600x/1) by NERC (Natural Environment Research Council, UK).

\section{REFERENCES}

[1] L.-K. Soh, et al, "ARKTOS: an intelligent system for SAR sea ice image classification," IEEE TGRS, vol. 42, no. 1, pp. 229,248, Jan. 2004.
[2] S. Salem, et al, "Image Segmentation by using threshold techniques," Int. J. of Computing, vol. 2, no. 5, May 2010.

[3] R. Banfield, "Ice floe identification in satellite images using mathematical morphology and clustering about principal curves," Journal of American Statistical Association, vol. 87, no. 417, pp. 7-16, 1992.

[4] X. Yang and D. A. Clausi, "SAR Sea Ice Image Segmentation Based on Edge-preserving Watersheds," in Fourth Canadian Conference on Computer and Robot Vision, Canada, 2007.

[5] J. Shen and Y. Xuezhi, "A new SAR sea ice image segmentation algorithm based on edge-preserving watershed," in 7th World Congress on Intelligent Control and Automation, WCICA, June 2008.

[6] R. Palenichka, et al, "Sea ice segmentation of SAR imagery using multi-temporal and multi-scale feature extraction," in $2^{\text {nd }}$ Int. Conf. on Space Technology (ICST), 2011.

[7] H. Bakir and M. Charfi, "Automatic medical image segmentation based on EPGV-Snake," in 6th International Multi-Conference on Systems, Signals and Devices, 2009.

[8] H. Deng and D. Clausi, "Unsupervised segmentation of synthetic aperture Radar sea ice imagery using a novel Markov random field model," IEEE TGRS, vol. 43, no. 3, pp. 528,538, March 2005.

[9] Q. Yu and D. Clausi, "SAR Sea-Ice Image Analysis Based on Iterative Region Growing Using Semantics," IEEE TGRS, vol. 45, no. 12, pp. 3919-3931, Dec. 2007.

[10] T. B. Ijitona, J. Ren, and P. B. Hwang, "SAR sea ice image segmentation using watershed with intensity-based region merging," in Proc. IEEE Int. Conf. on Computer and Information Technology (CIT), pp. 168-172.

[11] S. Sakhalkar, et al, "Effective SAR image segmentation and sea-ice floe distribution analysis via kernel graph cuts based feature extraction and fusion," in Proc. $4^{\text {th }}$ Int. Conf. on Sensor Networks Doctoral Consortium. INSTICC, 2015, pp. 28-37.

[12] C. Zhao, et al, "Improved sparse representation using adaptive spatial support for effective target detection in hyperspectral imagery,” Int. J. Remote Sensing, 34(24): 8669-8684, 2013.

[13] J. Han, et al, "Background prior based salient object deetction via deep reconstruction residual," IEEE Trans. CSVT, 2015.

[14] G. Cheng, et al, "Effective and efficient midlevel visual elements-oriented land-use classification using VHR remote sensing images," IEEE TGRS 53(8): 4238-49, 2015.

[15] J. Zabalza, et al, "Singular spectrum analysis for effective feature extraction in hyperspectral imaging," IEEE GRSL, 11(11): 1886-1890, 2014.

[16] J. Zabalza, et al, "Novel two-dimensional singular spectrum analysis for effective feature extraction and data classification in hyperspectral imaging," IEEE TGRS 53(8):4418-33, 2015. 